EPJ Web of Conferences 60, 16009 (2013)

DOI: $10.1051 /$ epjconf $/ 20136016009$

(C) Owned by the authors, published by EDP Sciences, 2013

\title{
Single top quark production in ATLAS at the LHC
}

\author{
Stillings, Jan A. ${ }^{1, a}$ on behalf of the ATLAS collaboration \\ ${ }^{1}$ Physikalisches Institut, Rheinische Friedrich-Wilhelms-Universität Bonn, Nußallee 12, 53115 Bonn, Germany
}

\begin{abstract}
Measurements of the single top quark production cross-sections in proton collisions with the ATLAS detector at the Large Hadron Collider are presented. The single top-quark production in the $t$ and $W t$-channels and the determination of the CKM matrix element $\left|V_{t b}\right|$ are discussed. A separate measurement of the top and antitop quark cross-sections and their ratio is shown. These measurements are sensitive to the parton distribution function in the proton. In addition, limits on exotic production in single top quark processes are outlined. This also includes the search for flavour-changing neutral currents and for additional $W^{\prime}$ bosons in the $s$-channel.
\end{abstract}

\section{Introduction}

At the Large Hadron Collider (LHC) top quarks can be produced via strong and electroweak interactions. While strong interactions lead to top-quark pair production, single top quarks are produced in electroweak interactions accompanied by other particles. The Standard Model of particle physics predicts the generation of single top quarks in three channels that have different initial and final states: the $t$-channel, the associated production of a $W$ boson and a top quark, referred to as $W t$-channel, and the $s$-channel. Examples for leading order Feynman diagrams of these are shown in Fig. 1. Theory predictions for the cross-sections at the LHC are summarised in Table 1.

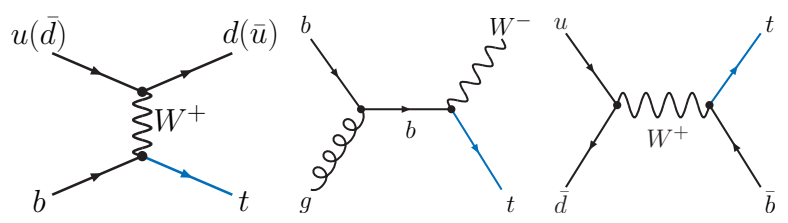

Figure 1. Leading order Feynman diagrams for single top quark production in the different channels: $t, W t$ and $s$.

\begin{tabular}{l|cc}
\hline Channel & $\sigma @ \sqrt{s}=7 \mathrm{TeV}$ & $\sigma @ \sqrt{s}=8 \mathrm{TeV}$ \\
\hline$t$-channel & $64.6_{-1.7}^{+2.6} \mathrm{pb}$ & $87.76_{-1.9}^{+3.4} \mathrm{pb}$ \\
$W t$-channel & $15.7 \pm 1.2 \mathrm{pb}$ & $22.4 \pm 1.5 \mathrm{pb}$ \\
$s$-channel & $4.6 \pm 0.2 \mathrm{pb}$ & $5.61 \pm 0.22 \mathrm{pb}$ \\
\hline
\end{tabular}

Table 1. Cross-sections of single top quark production in proton collisions for different collision energies based on NLO calculations from [1]

\footnotetext{
ae-mail: stillings@uni-bonn.de
}

There are interesting questions coupled to single topquark production, which can be addressed by measurements accomplished using proton collision data from the ATLAS detector [2]. The following document first presents measurements in the channels predicted by the Standard Model in Section 2 and later summarises the searches for new physics undertaken with single top quark topologies in Section 3.

\section{Standard Model verification}

The main interest in the single top-quark sector focusses on the isolation of the three channels for single top-quark production and the measurement of their respective crosssections. Deviations from the theory predictions could point to new physics, such as more than three fermion generations or alternative couplings. As all three production channels include a $W-t-b$ vertex, direct constraints of the Cabibbo-Kobayashi-Maskawa (CKM) matrix element $\left|V_{t b}\right|$ can be imposed. It is also possible to measure topand antitop-quark production cross-sections separately in the $t$-channel.

\section{$2.1 t$-channel measurements}

Production of single top quarks in the $t$-channel was the first channel to be observed by ATLAS using $156 \mathrm{pb}^{-1}$ of pp collision data at $\sqrt{s}=7 \mathrm{TeV}$. Using the larger amount of data collected in 2011 at $\sqrt{s}=7 \mathrm{TeV}$ and in 2012 at $\sqrt{s}=8 \mathrm{TeV}$ further measurements are possible.

An analysis using $1.04 \mathrm{fb}^{-1}$ of data of the 2011 run measures the production cross-section [4]. The selection requires one lepton with transverse momentum $p_{T}>$ $25 \mathrm{GeV}$ in the central region of the detector $(\eta<2.5$, where $\eta$ is the pseudorapidity) and a substantial amount of missing transverse energy $\left(E_{T}^{\text {miss }}>25 \mathrm{GeV}\right)$. It also demands two or three jets with $p_{T}>25 \mathrm{GeV}$ in an extended pseudorapidity range $(\eta<4.5)$ to account for the 
forward light-quark jet, which is special to the $t$-channel. Among the jets, at least one should be tagged as a $b$-quark jet. Additionally, a cut to reduce the impact of multijet channel is simultaneously applied on the transverse mass of the lepton- $E_{T}^{\text {miss }}$ system ${ }^{1}$ and the $E_{T}^{\text {miss }}: m_{T}(W)+E_{T}^{\text {miss }}>$ $60 \mathrm{GeV}$.

After enhancing single top quarks using the above event selection, two neural networks are trained to separate the signal from the background, one for each jet category. An example distribution of the neural network output for events with exactly 2 jets in shown in Fig. 2 . A clear signal excess can be observed in the bins with larger values of the discriminator. The distributions also give an impression of the main backgrounds. In the 2-jet category, the selected sample is dominated by $W+$ jets production, especially heavy-flavour jets. Other important backgrounds are the top-quark pair production, which becomes more important in the 3-jet category, and multijet production, which is estimated using data-based methods.

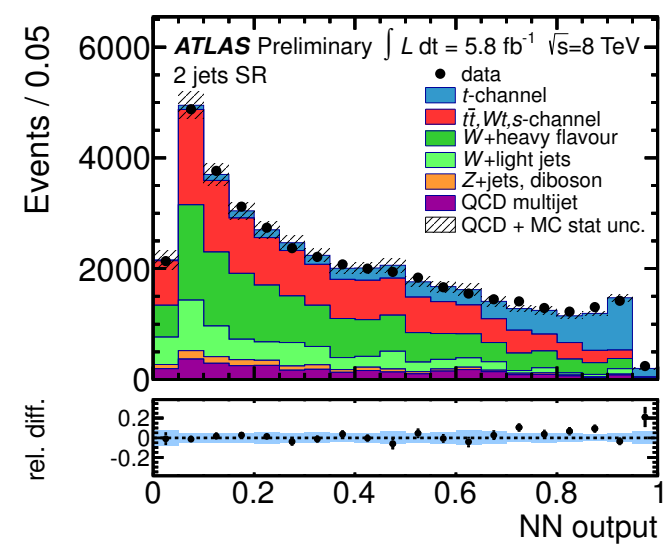

Figure 2. Neural network output distribution in the 2-jet category for the $\sqrt{s}=8 \mathrm{TeV}$ analysis. All component distributions are normalised to the result of the maximum-likelihood fit [5].

To extract the signal cross-section, a template fit implementing a binned likelihood is used. The measured crosssection is

$$
\sigma_{t}=83 \pm 4(\text { stat. })_{-19}^{+20} \text { (syst.) pb. }
$$

The significance of this measurement has been determined using pseudo-experiments and is expected to be $6 \sigma$ with $7.2 \sigma$ observed. The result is dominated by systematic uncertainties which mainly result from initial and final-stateradiation $(14 \%)$ and $b$-tagging efficiency $(13 \%)$.

Assuming a marginal contribution from the CKM matrix elements $\left|V_{t d}\right|$ and $\left|V_{t s}\right|$, it is possible to calculate $\left|V_{t b}\right|$ directly from the ratio of measured and predicted crosssections:

$$
\left|V_{t b}\right|=1.13_{-0.13}^{+0.14} \text { (exp.) } \pm 0.02 \text { (theo.) }
$$

\footnotetext{
${ }^{1} m_{T}(W)=\sqrt{\left(p_{T}(\ell)+E_{T}^{\text {miss }}\right)^{2}-\left(p_{x}(\ell)+E_{x}^{\text {miss }}\right)^{2}-\left(p_{y}(\ell)+E_{y}^{\text {miss }}\right)^{2}}$, for leptons $\ell$
}

An analysis using the same method has been performed using $5.8 \mathrm{fb}^{-1}$ of data collected in 2012 [5]. The event selection has been refined to the environment with higher collision energies. The measured cross-section is

$$
\sigma_{t}=95 \pm 2 \text { (stat.) } \pm 18 \text { (syst.) pb. }
$$

Again a calculation of $\left|V_{t b}\right|$ is executed and leads to a value of $\left|V_{t b}\right|=1.04_{-0.11}^{+0.10}$. Both cross-section and matrix element measurements show a good agreement with the Standard Model predictions within the estimated errors.

With the full dataset of the 2011 ATLAS run of $4.7 \mathrm{fb}^{-1}$ another analysis is able to measure the top quark and antitop quark cross-sections separately [6]. It uses very similar selection cuts as the $7 \mathrm{TeV}$ cross-section analysis in the lepton + jets channel described above. As the lepton stems from the decay of the $W$ boson which in turn is a product of the top-quark decay, its charge defines whether a top or an antitop quark was produced. The density of up quarks in the proton, given by the PDFs, is about twice as high as the down-quark density and is dominated by valence quarks at the characteristic momentum fractions, $x$, in the $t$-channel $(0.02,0.5)$ at the LHC energies. The ratio of the top quark and antitop quark cross-section is therefore sensitive to the ratio of the up-quark and down-quark PDFs.

The analysis sorts all events into two different categories based on the charge of the lepton. Four neural networks are used in the two and three-jet categories and for the different lepton charges to separate the signal from backgrounds. A simultaneous fit of the two jet-categories is then applied to obtain a result for each charge value. The measured cross-sections are

$$
\begin{aligned}
& \sigma_{t}(t)=53.2 \pm 1.7 \text { (stat.) } \pm 10.6 \text { (syst.) } \mathrm{pb} \text { and } \\
& \sigma_{t}(\bar{t})=29.5 \pm 1.5 \text { (stat.) } \pm 7.3 \text { (syst.) pb, }
\end{aligned}
$$

which are both in agreement with the Standard Model predictions. The main systematic uncertainty in this measurements comes from the jet energy scale with $20 \%$.

An estimation of the cross-section ratio leads to a value of

$$
R_{t}=1.81 \pm 0.10 \text { (stat.) }{ }_{-0.20}^{+0.21} \text { (syst.). }
$$

The impact of systematic uncertainties largely cancels as many factors are the same for both cross-section measurements. The result is statistically limited while the main systematic uncertainties follow from the background normalization $(5.5 \%)$ and the jet energy scale (4\%). A comparison with ratio calculations at next-to-leading order using different PDF sets shows agreement between measurement and the predictions as shown in Fig. 3. A summary of the presented results of $t$-channel production cross-sections is shown in Fig. 4.

\section{$2.2 W t$-channel measurements}

The production of single top quarks in the $t$-channel has been the first to be measured by ATLAS [3-5]. More recently the evidence of top-quark production in the $W t$ channel has been observed with an analysis that, using $2.05 \mathrm{fb}^{-1}$ of 2011 collision data, aims at isolating this 


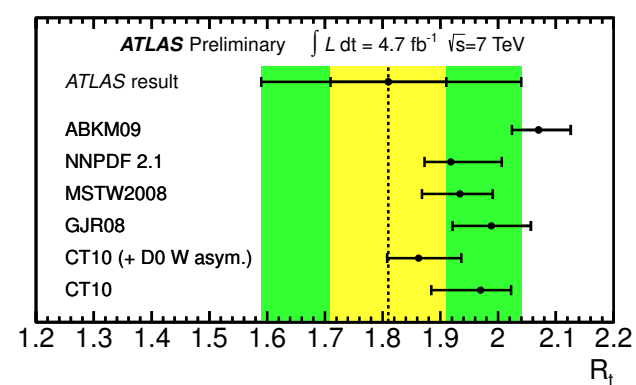

Figure 3. Calculated $R_{t}$ values for different NLO PDF sets. The error contains the uncertainty on the renormalisation and factorisation scales. The black line indicates the central value of the measured $R_{t}$ value. The combined statistical and systematic uncertainty of the measurement is shown in green, while the statistical uncertainty is represented by the yellow error band [6].

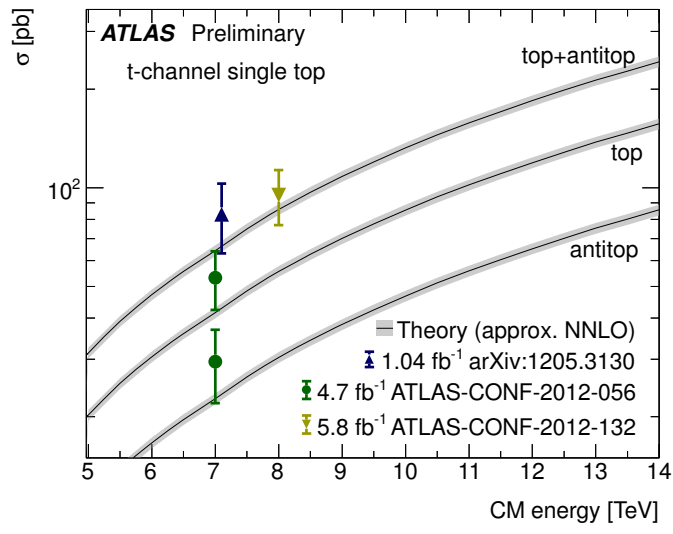

Figure 4. Summary of ATLAS $\sqrt{s}=7 \mathrm{TeV}$ and $\sqrt{s}=8 \mathrm{TeV}$ single top quark measurements in the $t$-channel [6].

channel [7]. The process involves the interaction of a gluon and a $b$-quark emitting an on-shell $W$ boson. Unlike the $t$-channel, the production in the $W t$-channel offers both a lepton + jets as well as a final state with two leptons in addition to the hardly accessible full-hadronic decay.

The dilepton channel offers the possibility for a very good veto of backgrounds which don't involve top quark production. To use this advantage a selection has been tailored to this channel, selecting only events with exactly two central oppositely-charged leptons with $p_{T}>25 \mathrm{GeV}$. At least one central jet with $p_{T}>30 \mathrm{GeV}$ is required. Although this jet should correspond to the $b$-quark jet from the top-quark decay, an additional $b$-tagging requirement has shown no advantage. $E_{T}^{\text {miss }}>50 \mathrm{GeV}$ is required to account for two neutrinos in the final state. Additional cuts are applied to veto events from $Z$-boson decays, which can lead to similar final states if accompanied by an additional jet.

The analysis uses the one-jet bin for the signal extraction as it offers the highest signal-to-background ratio. Higher jet multiplicities contain a larger contamination of top quark pairs and other backgrounds and are included to constrain their contribution. A boosted decision tree
(BDT) is used to form a single classifier as no single separating variables allow a signal isolation. It is trained using events from the one-jet bin and the output is computed for three categories: events with exactly one jet, events with two jets and events with three jets or more. The distribution for the one-jet category is shown in Fig. 5.

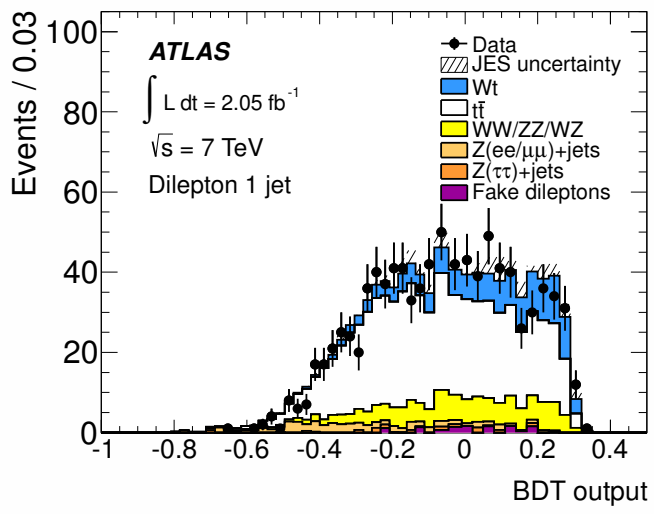

Figure 5. BDT output for selected events in the one-jet category. The $W t$ signal is normalised to the theory prediction [7].

A likelihood template fit is applied to extract the signal cross-section which is estimated to

$$
\sigma_{t}=16.8 \pm 2.9 \text { (stat.) } \pm 4.9 \text { (syst.) pb. }
$$

The result is compatible with the theory predictions and the main uncertainties come from statistics (17\%), jet energy scale systematics $(16 \%)$ and systematics related to the parton shower model $(15 \%)$. The significance of the measurement is expected to be $3.4 \sigma$ while $3.3 \sigma$ is observed.

In the same way as for the analyses above, the matrix element $\left|V_{t b}\right|$ has been calculated to $\left|V_{t b}\right|=1.03_{-0.19}^{+0.16}$. This analysis marks the first evidence for single top-quark production in the $W t$-channel.

\section{Searches for new physics}

The topologies of single top-quark production offer a variety of possibilities to search for new physics beyond the Standard Model. While cross-section measurements are rather model-independent probes, several ATLAS analyses search for specific manifestations of new physics. Four examples are described in the following.

\subsection{Search for $W^{\prime}$ production}

An analysis using $14.3 \mathrm{fb}^{-1}$ of 2012 collision data investigates the existence of new heavy charged gauge bosons [8], which are predicted by a variety of theoretical models and are usually called $W^{\prime}$. They can potentially be observed in $t$ - $b$-resonances which can be probed in the $s$ channel single top quark topology. In the $s$-channel diagram in Fig. 1, the $W$ boson is replaced by a $W^{\prime}$. Such a search is very sensitive to lepto-phobic $W^{\prime}$ models and can complement leptonic searches in e.g. $W+$ jets topologies. 
The analysis probes both right-handed and left-handed models. The selection requires one central lepton, $E_{T}^{\text {miss }}$ and two or three jets in the central detector region, two of which are $b$-tagged. A BDT is trained on a reference simulation sample that models a heavy boson with a mass of $M_{W^{\prime}}=1.75 \mathrm{TeV}$. The BDT output classifier is also calculated for a variety of other masses which is shown exemplified in Fig. 6. As no excess if found, limits of $M_{W_{L}^{\prime}}>1.74 \mathrm{TeV}$ and $M_{W_{R}^{\prime}}>1.84 \mathrm{TeV}$ are extracted at the $95 \%$ confidence level (C.L.) for the masses of the lefthanded and right-handed $W^{\prime}$ bosons respectively.

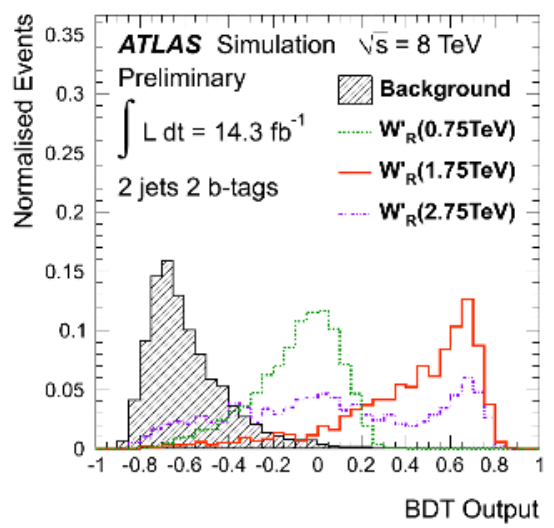

Figure 6. Expected BDT output distributions for the background and several $W_{R}^{\prime}$-boson processes in the 2-jet signal region. All distributions are normalised to unit area [8].

\subsection{Search for $b^{*}$ production}

The 2011 dataset corresponding to $4.7 \mathrm{fb}^{-1}$ is used to search for strong production of excited quarks which decay via electroweak processes into a top quark and a $W$ boson [9]. The $b$ quark propagator in the Feynman diagram of the $W t$-channel in Fig. 1 is replaced by a $b^{*}$ propagator in this case and the search uses a similar selection. The analysis uses both single and dilepton final states to probe for a potential signal. The dilepton variant uses $H_{T}$ as discriminating variable, which is defined as the sum of $p_{T}$ of the two leptons and the leading jet. In the single lepton final state, the reconstructed invariant mass of the $W t$ system is probed. The single expected neutrino in the final state can be reconstructed from $E_{T}^{\text {miss }}$ and a $W$-boson mass constraint. The distribution of the variable compared to an expected signal for an excited $b$ quark with $800 \mathrm{GeV}$ mass is presented in Fig. 7.

As no excess of signal has been observed in the distributions, a template fit has been used to extract a variety of limits for new physics models including an excited bottom quark. It excludes $b^{*}$ quarks with unit size chromomagnetic coupling and Standard Model-like electroweak coupling up to masses of $870 \mathrm{GeV}$ at $95 \%$ confidence level.

\subsection{FCNC in single top quark production}

In the Standard Model, flavour-changing neutral current (FCNC) processes are forbidden at tree level and sup-

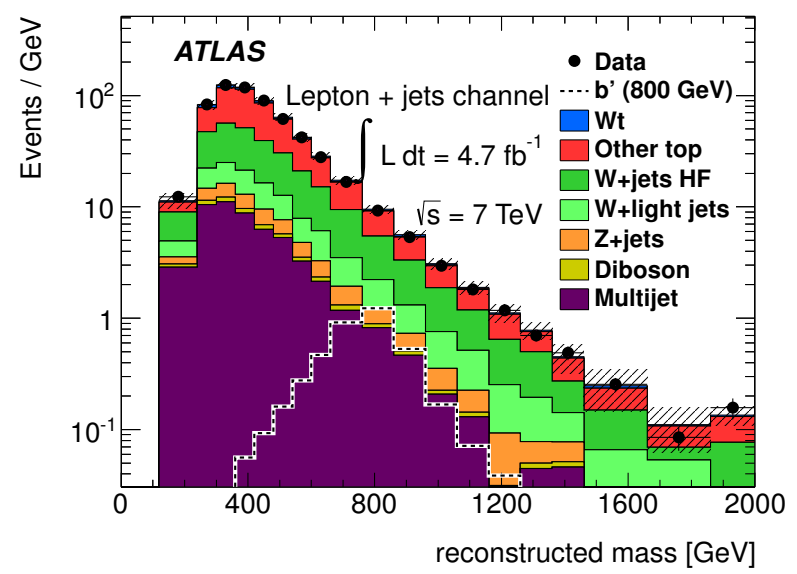

Figure 7. Reconstructed mass distributions for data and background expectation for the lepton+jets channel. The hatched band shows the uncertainty due to the background normalisation. The signal for a mass of $800 \mathrm{GeV}$ is also shown. The last bin includes overflows [9].

pressed at higher orders. Many new physics models like MSSM, R-parity violating supersymmetry and the twoHiggs doublet model predict higher rates of FCNC which could be measured in single top-quark production. An analysis at ATLAS using $2.05 \mathrm{fb}^{-1}$ of data from the 2011 run searches for such processes measuring the process shown in Fig. 8 [10].

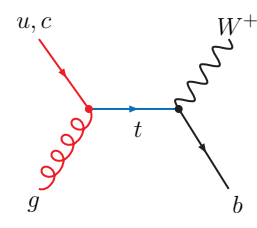

Figure 8. Feynman diagram for the single top-quark production via flavour-changing neutral currents.

The analysis uses the same selection as the $t$-channel cross-section measurement, but requires one jet in the final state only, which has to be $b$-tagged. Two neural networks are trained separately in the electron and muon channels using simulated samples of FCNC events and the expected backgrounds. Figure 9 shows the output distributions scaled to the number of observed events.

The background events describe the data accurately and there is no excess observed. An upper limit on the cross-section of possible FCNC in the top-quark production of $\sigma<3.9 \mathrm{pb}$ at the $95 \%$ confidence level is extracted using a Bayesian statistical analysis. This is interpreted as limits on the coupling constants that are assumed for the top quark production vertices and finally in terms of branching fractions of the processes $t \rightarrow c g$ and $t \rightarrow u g$. The analysis substantially improves the best limits set so far by the D0 experiment [11].

\subsection{Probe for anomalous couplings}

Finally an analysis using again $4.7 \mathrm{fb}^{-1}$ of 2011 proton collision data searches for anomalous couplings in the $t$ channel single top-quark production [12], which changes 


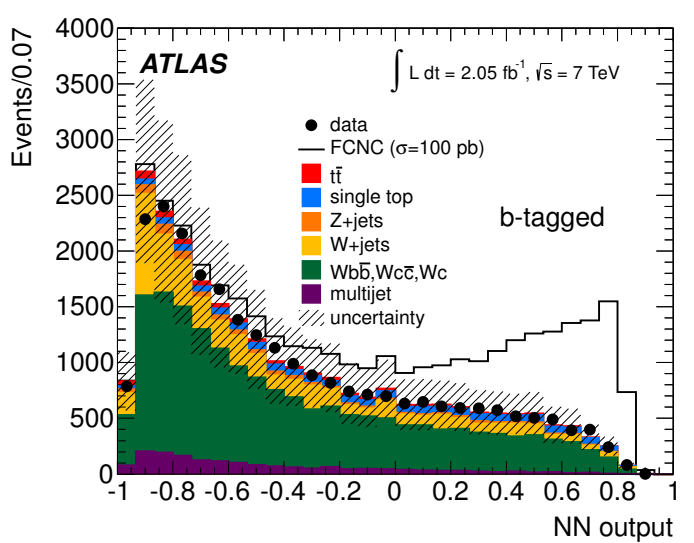

Figure 9. Neural network output distribution scaled to the number of observed events in the $b$-tagged sample. The signal contribution is shown stacked on top of the backgrounds normalised to $100 \mathrm{pb}$. The hatched band indicates the statistical uncertainty from the sizes of the simulated samples and the uncertainty in the background normalisation [10].

the coupling at the $W-t-b$ vertex in the diagram in Fig. 1. In the $t$-channel, top quarks are expected to be highly polarised along the spectator quark's direction $(P \approx 0.9)$. Based on the top-quark spin $\left(\vec{s}_{t}\right)$ a transverse $(\vec{T})$ and a normal $(\vec{N})$ reference direction can be defined in the top quark rest frame helicity basis:

$$
\vec{N}=\overrightarrow{s_{t}} \times \vec{q} \quad \text { and } \quad \vec{T}=\vec{q} \times \vec{N},
$$

where $\vec{q}$ is the $W$ boson momentum in the helicity basis. It is then possible to define angles between the lepton in the $W$ boson rest frame and the two new directions $\left(\theta^{N}\right.$ and $\left.\theta^{T}\right)$.

Following the standard $t$-channel event selection detailed before, the analysis searches for an asymmetry in the distribution of the angle $\theta^{N}$ which is defined as

$$
A_{\mathrm{FB}}^{N}=\frac{N_{\mathrm{evt}}\left(\cos \theta^{N}>0\right)-N_{\mathrm{evt}}\left(\cos \theta^{N}<0\right)}{N_{\mathrm{evt}}\left(\cos \theta^{N}>0\right)+N_{\mathrm{evt}}\left(\cos \theta^{N}<0\right)} .
$$

This asymmetry is very sensitive to the imaginary part of the anomalous right-handed tensor coupling $g_{R}$, whose existence would point at charge-parity $(C \mathcal{P})$ violation in the top-quark decay.

The observed distribution of $\cos \theta^{N}$ for the electron channel is shown in Fig. 10 and the resulting asymmetry is measured as

$$
A_{\mathrm{FB}}^{N}=0.031 \pm 0.065 \text { (stat.) }{ }_{-0.031}^{+0.029} \text { (syst.). }
$$

For small values of $g_{R}$ and the Standard Model predictions $V_{L}=1$ and $V_{R}=g_{L}=0$ for the vector and tensor couplings, the imaginary part of $g_{R}\left(\mathbb{I}\left(g_{R}\right)\right)$ can be calculated from $A_{\mathrm{FB}}^{N}=0.64 P \mathbb{I}\left(g_{R}\right)$ [13] which allows the computation of a limit on its value:

$$
\mathbb{I}\left(g_{R}\right)=[-0.20,0.30] @ 95 \% \text { C.L. }
$$

All the results are consistent with Standard Model expectation.

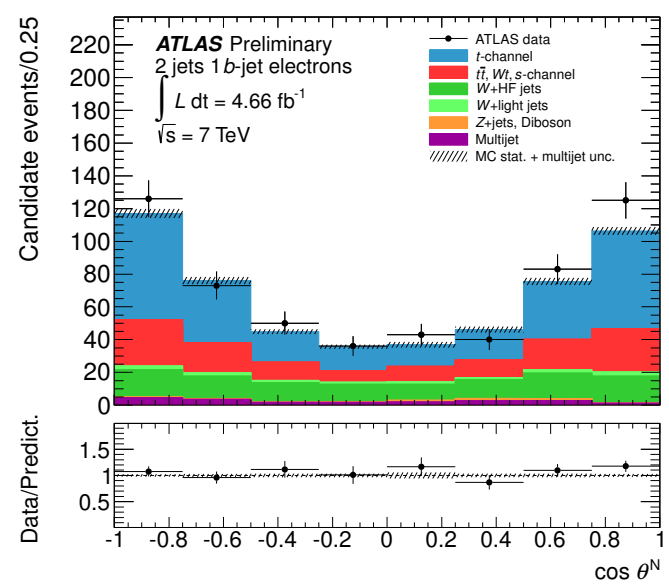

Figure 10. Reconstructed $\cos \theta^{N}$ distribution obtained at selection level for the electron channel. The uncertainties shown on the prediction take into account MC statistics and the $50 \%$ systematic uncertainty on the normalization of the multijet background [12].

\section{Summary and outlook}

Using data from proton collisions at $\sqrt{s}=7 \mathrm{TeV}$ and $8 \mathrm{TeV}$, ATLAS has performed a rich analysis program using single top-quark topologies. Events from $t$-channel production can be extracted with high significance and several cross-section measurements have been performed. Evidence has been seen for the $W t$-channel production and a limit has also been set on the $s$-channel [14], which is not covered in this proceeding. Single top quarks offer the possibility to search for new physics. Limits could be set on the FCNC in the top quark production and on $C \mathcal{P}$ violation in its decay as well as on the production of exotic $b^{*}$ quarks and $W^{\prime}$ bosons. Many new analyses are in preparation using the full statistics of the $8 \mathrm{TeV}$ collision dataset and also addressing the systematic uncertainties which impose the largest limitations in many of the presented analyses.

\section{References}

[1] N. Kidonakis, Phys. Rev. D, 83, 091503 (2010); 82, 054018 (2010); 81, 054028 (2010)

[2] ATLAS Collab., JINST, 3, S08003 (2008)

[3] ATLAS Collab., ATLAS-CONF-2011-088, (2011)

[4] ATLAS Collab., Phys. Lett. B, 717, 330 (2012)

[5] ATLAS Collab., ATLAS-CONF-2012-132, (2012)

[6] ATLAS Collab., ATLAS-CONF-2012-056, (2012)

[7] ATLAS Collab., Phys. Lett. B, 716, 142 (2012)

[8] ATLAS Collab., ATLAS-CONF-2013-050, (2013)

[9] ATLAS Collab., Phys. Lett. B, 721, 171 (2013)

[10] ATLAS Collab., Phys. Lett. B, 712, 351 (2012)

[11] D0 Collab., Phys. Lett. B, 693, 81 (2010)

[12] ATLAS Collab., ATLAS-CONF-2013-032, (2013)

[13] J.A. Aguilar-Saavedra and J. Bernabéu, Nucl. Phys. B, 840349 (2010)

[14] ATLAS Collab. ATLAS-CONF-2011-118, (2011) 